\title{
Stochastic Reachable Sets of Interacting Traffic Participants
}

\author{
Matthias Althoff, Olaf Stursberg, and Martin Buss
}

\begin{abstract}
Knowledge about the future development of a certain road traffic situation is indispensable for safe path planning of autonomous ground vehicles or action selection of intelligent driver assistance systems. Due to a significant uncertainty about the future behavior of traffic participants, the prediction of traffic situations should be computed in a probabilistic setting. Under consideration of the dynamics of traffic participants, their future position is computed probabilistically by Markov chains that are obtained with methods known from hybrid verification. The characteristic feature of the presented approach is that all possible behaviors of traffic participants are considered, allowing to identify any dangerous future situation. The novel contribution of this work is the explicit modeling of the interaction of traffic participants, which leads to a more accurate prediction of their positions. Results are demonstrated for different traffic situations.
\end{abstract}

\section{INTRODUCTION}

A general trend in automotive research is the detection of the vehicle environment in order to restrict, advice or automate driving maneuvers to improve driving safety and comfort. Exemplary systems using information about surrounding traffic participants are, e.g. adaptive cruise control, lane change assistance, collision mitigation and avoidance [1], [2], [3]. The knowledge about the actual traffic situation becomes even more important in the future, when more sophisticated driver assistance systems will be developed. Especially for current research in the field of fully autonomous driving, knowledge of the traffic situation is crucial. In order to plan or restrict driving maneuvers of intelligent driver assistance systems or fully autonomous cars, it is not sufficient to only have knowledge about the current situation. The more advanced the systems become, the more important is a prediction of the traffic situation. Assume a situation in which a dynamic obstacle suddenly appears in front of a car. A prediction of the motion of objects in the environment can support the decision whether changing lanes or immediate braking (possibly taken a collision with the obstacle at reduced speed into account) is a better option.

However, measured data (e.g. position, velocity, ...) from other traffic participants is subject to uncertainties. Additionally, the future behavior of other traffic participants is barely known. Due to the high level of uncertainty, it is not sufficient to consider single driving maneuvers but multiple possibilities must be evaluated, e.g. by using Monte Carlo techniques in [4], [5], [6]. The advantage of these techniques is that complicated highly nonlinear vehicle dynamics can be considered, but the techniques usually require many

All authors are with the Institute of Automatic Control Engineering (LSR), Technische Universität München, 80290 München, Germany. \{althoff, stursberg, mb\}@tum.de simulation runs for suitably capturing the possible evolutions. Nevertheless, these methods can, in principle, not cover all possible behaviors (except of very simple configurations). In contrast, methods for algorithmic verification can guarantee to detect any dangerous situation. The basic principle of algorithmic verification of continuous and hybrid systems is as follows: First, the set of all possible states which a system can reach is computed starting from a set of initial conditions for a given set of input trajectories. Second, the reachable set is intersected with regions in the state space that are unsafe. If the intersection is empty, the system is safe. For an online verification algorithm operating in a vehicle, unsafe sets are road areas which are occupied by surrounding traffic participants. Verification techniques can be extended to stochastic model components, what means that the probability for reaching a given set is obtained in addition. Stochastic verification techniques have been investigated to increase air traffic safety in [7], [8]. In previous works of the authors, an online method for stochastic verification of road traffic scenarios has been developed [9], [10].

In order to obtain sound verification results for traffic scenarios that involve several traffic participants, their possible interactions should be considered. For example, if for a chain of cars, the vehicle driving in front decelerates, it is likely that the following cars brake. If braking would not be enforced, the car following the braking car would share equal road regions with high probability after a certain time interval. Thus, negligence of interaction leads to wrong probability distributions, where road regions may be occupied by traffic participants with a computed probability greater than one. Interactions between traffic participants are considered in microscopic traffic simulations, such as in e.g. [11], [12]. However, microscopic traffic simulations take only single driving behaviors into account. Applying Monte Carlo techniques to simulations which consider interaction between traffic participants results in a fast growing tree of possible situations to be considered [13]. For instance, if a vehicle is considered by $n$ simulations for a certain time interval, one would have to compute $n^{2}$ simulations for possible combinations of behaviors for two vehicles (if all combinations were possible). At last, there is no or very little work on threat assessment by considering interacting traffic participants. With respect to the context of stochastic verification of traffic situations, the consideration of interacting objects has not yet been considered, to the authors' knowledge. Thus, the novel contribution of this work is to propose a method allowing to compute stochastic reachable sets of interacting traffic participants. The proposed approach is efficient in the sense that it can be applied online. 


\section{Problem Statement and ObJective}

Given the probability distribution of positions and velocities of traffic participants on a section of a road network, the objective is to compute the approximative probability distribution of the position of the traffic participants for a limited predicted horizon. The values of the initial states (position, velocity) of the traffic participants and their possible inputs (acceleration values) are restricted to bounded sets. This restriction is considered by computing the probability distributions in a way, in which the possible states of each traffic participant are guaranteed to stay within the region of nonzero probability. This region together with the probability distribution is referred to as the stochastic reachable set. In contrast to the overapproximative computation of the region of non-zero probability (i.e. no trajectory leaves this region), the probability distribution within this region is computed approximatively ${ }^{1}$.

The stochastic reachable sets of traffic participants are used to evaluate the safety of planned paths of autonomous cars by checking if it is likely to drive into a reachable set of another traffic participant. If the reachable set of the autonomous car does not intersect any of the reachable sets of other traffic participants, one can guarantee that the planned path is safe. The situation in Fig. 1(a) is not safe, as the stochastic reachable sets for the second time interval $\tau_{i+2}=\left[t_{i+1}, t_{i+2}\right]$ of both vehicles intersect. The prediction of future traffic situations is limited to a time horizon $t_{f}$ and repeated in any discrete time $t_{i}$ using a prediction horizon $\left[t_{i}, t_{i}+t_{f}\right]$, see Fig. 1(b).

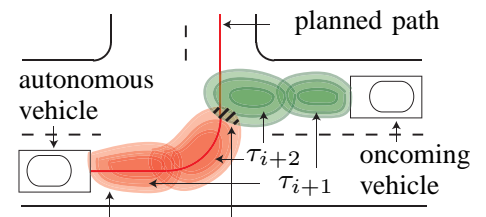

stochastic intersection reachable set

(a) Exemplary traffic situation

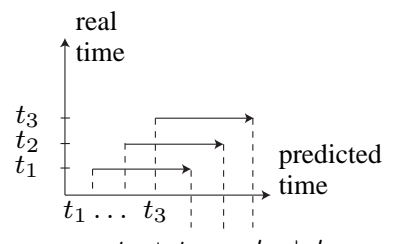

$t_{1}+t_{f} \ldots t_{3}+t_{f}$

(b) Predicted time horizon
Fig. 1. Illustration of reachable sets and the resulting prediction horizon.

The computation of the probability distribution of the future position of traffic participants is based on the assumption that traffic regulations are met. This excludes behaviors such as vehicles crashing into other vehicles on purpose, or that vehicles are driving backwards on a lane. However, if such behavior is observed by the autonomous car, the stochastic reachable set has to be computed based on all possible behaviors considering the possible set of steering and acceleration inputs. This work considers only permitted behavior, although the stochastic reachable set obtained by regarding all possible behaviors, can be computed by the same approach. In order to focus on the new aspect of the interaction between traffic participants, the autonomous car

\footnotetext{
${ }^{1}$ An overapproximative computation of the probability distribution would not be a probability distribution anymore as the overall integral would be greater than one.
}

is not explicitly considered in the sequel. The autonomous car is just a special case of a traffic participant as its planned trajectory is known within the prediction horizon.

\section{Modeling CONCEPT OF TRAFFic PARTiCiPANTS}

The motion of traffic participants is modeled in two stages. First, the paths which a vehicle can follow are generated. They do naturally arise from the possible routes that the vehicle can take in the examined road network segment, see Fig. 2(a) for a T-intersection. These paths are broken up into equidistant segments. Besides the possible paths of a vehicle, the deviation from these paths is modeled by a static piecewise constant probability distribution that varies between road user types. Examples for these probabilities are given for a car/truck on the left and a bicycle on the right lane of a road in Fig. 2(b). The variable $\Delta s$ denotes the length of the path segments, $\delta$ the deviation from the paths and $p(s, \delta)$ the value of the probability density distribution of the position of the vehicle.

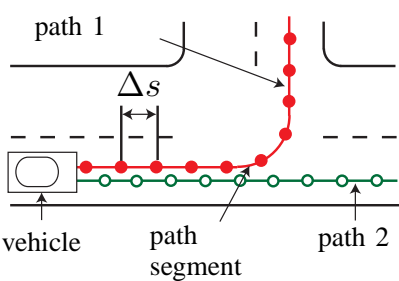

(a) Possible paths of a vehicle

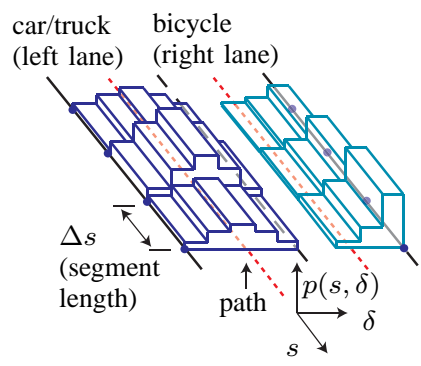

(b) Deviation examples from a path
Fig. 2. Path generation and deviation of traffic participants.

\section{A. Dynamic Model of a Single Vehicle}

In a second step, the longitudinal dynamics of the vehicles along the paths is considered. The dynamics is modeled by a hybrid automaton [14] which combines discrete and continuous dynamics. The discrete dynamics is modeled by four modes: acceleration, deceleration, standstill and speed limit. The mode changes from deceleration to standstill if the velocity of the vehicle is zero and from acceleration to speed limit, if the vehicle has reached the speed limit. The mode acceleration is active, if the acceleration command $u \in$ $[-1,1]$ of the driver is positive or equal zero $(u \in[0,1])$ and the deceleration mode is active if $u$ is negative $(u \in[-1,0[)$, see Fig. 3. The value $u=-1$ represents full braking, $u=1$ full acceleration, and the continuous dynamics is modeled as:

$$
\dot{s}=v, \quad \dot{v}=f_{d}(v, u)
$$

where $s$ is the position on the path and $v$ is the velocity of the vehicle. The dynamics of the speed $f_{d}(v, u)$ depends on the discrete mode $d \in\{1,2,3,4\}$ which are numbered in Fig. 3:

$$
f_{1}(v, u)=c_{1}\left(1-\left(v / c_{2}\right)^{2}\right) u, \quad f_{2}=f_{4}=0, \quad f_{3}(u)=c_{1} u
$$


and $c_{1}, c_{2}$ are vehicle specific constants which vary for trucks, cars and bicycles. The models for the system dynamics are exemplary and can be exchanged by other nonlinear models. For pedestrians, the split-up in path generation and path following does only make sense for designated paths which cross a road (e.g. crosswalks). In other cases, an approach for human motion in free space has to be used.

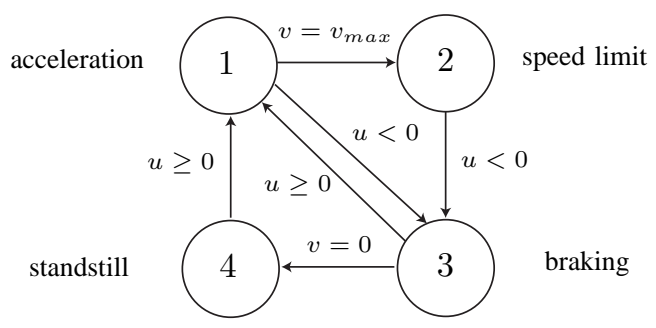

Fig. 3. Hybrid vehicle dynamics.

\section{B. Interaction between two Vehicles on a Lane}

In order to study interaction between two vehicles on a lane, one has to consider two instances of the hybrid vehicle model. The scenario of two vehicles driving on a lane is regarded as the archetype of vehicle-to-vehicle interaction, from which more complicated scenarios like an intersection scenario can be built, as shown later. The two investigated vehicles are referred to as vehicle $\mathrm{A}$ and vehicle $\mathrm{B}$, where A follows $\mathrm{B}$ on the same lane. The position and velocities are indexed as $s^{A}, v^{A}$ for car $\mathrm{A}$ and $s^{B}, v^{B}$ for car B. The distance $d$ between both cars is computed as $d=s^{B}-s^{A}-L$ where $L$ is the distance of the centers of mass of both cars when they stand bumper-to-bumper $\left(s^{A}, s^{B}\right.$ refer to the position of the center of mass).

The goal of the scenario is to determine if a constant acceleration command $u^{A}$ of car $\mathrm{A}$ for a time interval $t \in[0, T]\left(T \in \mathbb{R}^{+}\right)$results in an accident for a given $u^{B}$, where the initial velocity and position of both vehicles are known. Therefore, the hybrid model of car A and B are simulated for a time interval $t \in[0, T]$. Next, it is computed whether car A can avoid a crash if car B fully brakes at time $t=T$. Due to the reaction time $\delta$ of vehicle $\mathrm{A}$, it is further simulated for $t \in[T, T+\delta]$ with the previous acceleration command $u^{A}$, where vehicle $\mathrm{B}$ is simulated for $u^{B}=-1$. Next, both vehicles are simulated for $u^{A}=u^{B}=-1$ until car $\mathrm{A}$ is in mode standstill which happens at time $t_{s}$. The acceleration command $u^{A}$ is safe for $t \in[0, T]$ if the distance is positive $(d>0)$ for $t \in\left[0, t_{s}\right]$. In case, that $\dot{v}^{B} \leq \dot{v}^{A}$ for $u^{A}=u^{B}=-1$, it is sufficient to check the distance at the final time $\left(d\left(t_{s}\right)>0\right)$ to determine whether a crash can occurr. The information, whether a crash occurs on a lane for a given situation of two vehicles, is used later for the computation of stochastic reachable sets of interacting traffic participants.

\section{Computing Stochastic Reachable Sets Using MARKOV CHAINS}

So far, the model for a traffic participant has been set up. It remains to compute the stochastic reachable set of a traffic participant, given its initial probability distribution. In this work, stochastic reachable sets are computed by Markov chains, where each chain approximates the behavior of a single traffic participant. Markov chains can be built for different types of traffic participants, such as trucks, cars, bicycles and pedestrians. E.g. for a traffic scene with one truck and two cars, one Markov chain for the truck, and two Markov chains for the two cars would be instantiated.

\section{A. Approximating Uncertain Hybrid Dynamics by Markov Chains}

Markov chains are dynamic systems with a discrete state $z=i \in\{1, \ldots, l\}$ where for each time point only the probability distribution ${ }^{2} p_{i}=P(z=i)$ of the possible states is known. For discrete time Markov chains, as they are used in this work, the probability distribution for the next time point depends only on the probability distribution of the current time. Probability distributions of neighbored time points $t_{k}=k T$ and $t_{k+1}=(k+1) T\left(k \in \mathbb{N}^{+}, T \in \mathbb{R}^{+}\right.$ is the time increment) are connected via a transition matrix $\Phi: p((k+1) T)=\Phi p(k T)$. The discrete states of the Markov chains for the road vehicles are obtained by discretizing the original continuous state space into cells. Thus, each discrete state of the Markov chain represents a certain region of the originally continuous state space of the traffic participants. The discretization of the continuous state space of a hybrid system, as well as the approximation of its dynamics by a Markov chain is performed offline as presented in [9], [10]. The state space is discretized rectangularly and equidistantly so that all cells $X_{i}$ are interval hulls of equal size: $X_{i}=$ ]$\left.\underline{x}_{i}, \bar{x}_{i}\right], \quad \bar{x}_{i}-\underline{x}_{i}=c, \quad \underline{x}_{i}, \bar{x}_{i}, c \in \mathbb{R}^{n}$. The index $i$ refers to the number of the discrete state $z$ that the cell is associated with. In particular, for a vehicle driving on a path, each index $i$ represents a two-dimensional cell, where one dimension represents a segment on the path and the other dimension a velocity interval. It is once more noted that the one-dimensional value of the position on the path is sufficient to obtain the probability distribution of the vehicle in two-dimensional space, because the deviation probability distribution is equal for all path segments (see Sec. III). Besides the state space, the continuous input $u$ of the system is also equidistantly discretized. The cells of the input space are denoted $U_{j}$ and $j$ refers to a discrete input mode, such that $u \in U_{j}$ if the discrete input $y=j$ is active. In case of the traffic scenario, each input interval $U_{j}$ represents an acceleration interval of a vehicle. The transition matrix which approximates the original hybrid dynamics for a time step with input $j$ is denoted by $\Phi^{j}(T)$ and $\Phi^{j}([0, T])$ for a time interval. The discrete probability vector that represents the stochastic reachable set at a time point $t=k T$ is denoted by $p(k T)$ and the one representing a time interval $t \in[(k-1) T, k T]$ is denoted by $p([(k-1) T, k T])$. Both probability vectors are computed as:

$$
\begin{aligned}
p((k+1) T) & =\Phi^{j}(T) p(k T), \\
p([k T,(k+1) T]) & =\Phi^{j}([0, T]) p(k T),
\end{aligned}
$$

${ }^{2} P()$ is the operator returning the probability of an event 
for $y=j$ in $t \in[k T,(k+1) T]$. Note that $p((k+1) T)$ and $p([k T,(k+1) T])$ are computed based on the probability vector of the previous time point $p(k T)$. In many cases, not only the system state, but also the system input interval $y$ is uncertain: the probability vector $q$ for the input is defined analogously to $p$ by $q_{j}=P(y=j)$. The modified computation of the probability distribution of the Markov chain is:

$$
\begin{aligned}
p((k+1) T) & =\sum_{j} \Phi^{j}(T) q_{j}([k T,(k+1) T]) p(k T), \\
p([k T,(k+1) T]) & =\sum_{j} \Phi^{j}([0, T]) q_{j}([k T,(k+1) T]) p(k T) .
\end{aligned}
$$

The probability distribution $q_{j}([k T,(k+1) T])$ is assumed to be constant for one time interval. In the following, it is presented how the probability distribution $q_{j}([k T,(k+1) T])$ of the acceleration input of a vehicle varies based on the own state distribution and the state and input distribution of the vehicle driving ahead.

\section{B. Interaction between two Traffic Participants}

In this subsection, the interaction between two vehicles, modeled as Markov chains, is considered. The vehicles are referred to as vehicle $A$ and $B$, analogously to Sec. III-B. The interaction is performed by obtaining the input probability $q^{A}$ of vehicle $A$ based on the probability vector $p^{A}, p^{B}$ of vehicles $A, B$ and the input probability $q^{B}$ of vehicle $B$. In other words, the probability distribution of the acceleration intervals of the following vehicle is determined based on the current state of both vehicles and the acceleration behavior of the vehicle driving in front. Thus, the aim is to calculate the conditional probability $P\left(y^{A}=j \mid y^{B}=m, z^{A}=i, z^{B}=\right.$ $o$ ). The conditional probabilities for $y^{A}$ are obtained, based on the number of collision-free inputs $y^{A}$, when $z^{A}=i$, $z^{B}=o$ and $y^{B}=m$ are given. The number of collision free inputs is denoted by $h$. It is clear, that the $h$ collision free inputs are those that exhibit the highest negative acceleration. The probabilities for the inputs which result in a collision are set to zero and the remaining non-zero probabilities are stored in a look-up table based on heuristics or on results obtained from driving experiments. For example, the action of heavy braking is chosen with a higher probability, if heavy acceleration is not allowed, compared to a situation in which it is allowed. Further heuristics that may be applied are that full braking and full acceleration is unlikely (unless it is the only option) and that it is more likely that vehicles accelerate until speed-limit rather than decelerating. The general form of the look-up table is given in table I: the discrete input probability $q_{1}^{A}$ in table I represents the probability for strongest possible braking and $q_{e}^{A}$ for highest possible acceleration. For the values in between, the acceleration is gradually increased. It is emphasized that a change in the values of the non-zero probabilities in the look-up table does only affect the probability distribution within the region of non-zero probability. The region of non-zero probability is unaffected, such that a collision free trajectory of the
TABLE I

INPUT PROBABILITIES BASED ON THE NUMBER OF COLLISION-FREE INPUTS.

\begin{tabular}{l|llll}
\hline $\begin{array}{l}\text { collision-free } \\
\text { acceleration intervals } h\end{array}$ & $q_{1}^{A}$ & $q_{2}^{A}$ & $\ldots$ & $q_{e}^{A}$ \\
\hline$e$ & $\neq 0$ & $\neq 0$ & $\ldots$ & $\neq 0$ \\
$e-1$ & $\neq 0$ & $\neq 0$ & $\ldots$ & 0 \\
$\vdots$ & & & & \\
1 & 1 & 0 & $\ldots$ & 0 \\
0 & 1 & 0 & $\ldots$ & 0 \\
\hline
\end{tabular}

autonomous car (i.e. the non-zero probability region is not entered) can be guaranteed even if the probability values of the look-up table differ from the exact values.

The number $h$ of acceleration intervals leading to a possible accident are obtained by a worst case analysis. For vehicle A, the maximum possible values for position, velocity and acceleration within the cells of the discrete states and inputs are chosen. For the leading vehicle B, the minimum values are selected:

$$
\begin{aligned}
& {\left[\begin{array}{l}
s^{A}(0) \\
v^{A}(0)
\end{array}\right]=\max \left(X_{i}\right), \quad u^{A}=\max \left(U_{j}\right),} \\
& {\left[\begin{array}{l}
s^{B}(0) \\
v^{B}(0)
\end{array}\right]=\min \left(X_{o}\right), \quad u^{B}=\min \left(U_{m}\right),} \\
& \text { for } z^{A}=i, \quad z^{B}=o, \quad y^{A}=j, \quad y^{B}=m
\end{aligned}
$$

The result whether a crash occurs, is computed as previously described in Sec. III-B, using the above presented worst case initial conditions. As mentioned in Sec. III-B, the reaction time of traffic participants is also taken into account.

For further computations, the conditional probabilities $P\left(y^{A}=j \mid y^{B}=m, z^{A}=i, z^{B}=o\right)$ for all combinations of discrete states and inputs of vehicle $A$ and $B$ are stored in an $j \times m$ array of $i \times o$ matrices denoted as $\Omega_{i o}^{j m}$, such that

$$
\Omega_{i o}^{j m} \hat{=} P\left(y^{A}=j \mid y^{B}=m, z^{A}=i, z^{B}=o\right) .
$$

This matrix array is also referred to as the array of interaction matrices. It is noted that the array of interaction matrices is computed offline. Further, the joint probability $P\left(y^{B}=m, z^{B}=o\right)$ is denoted $p_{o}^{B, m}$ and events such as $z^{A}=i$ are abbreviated by $z_{i}^{A}$ in the following to achieve a concise notation for the next derivations. The joint probability $P\left(y_{j}^{A}, y_{m}^{B}, z_{i}^{A}, z_{o}^{B}\right)$ is obtained as:

$$
P\left(y_{j}^{A}, y_{m}^{B}, z_{i}^{A}, z_{o}^{B}\right)=P\left(y_{j}^{A} \mid y_{m}^{B}, z_{i}^{A}, z_{o}^{B}\right) P\left(y_{m}^{B}, z_{i}^{A}, z_{o}^{B}\right) .
$$

It is further assumed that $P\left(y_{m}^{B}, z_{o}^{B}\right)$ and $P\left(z_{i}^{A}\right)$ are independent such that $P\left(y_{m}^{B}, z_{i}^{A}, z_{o}^{B}\right)=P\left(y_{m}^{B}, z_{o}^{B}\right) P\left(z_{i}^{A}\right)$. This assumption is a good approximation in practice as $P\left(y_{m}^{B}, z_{o}^{B}\right)$ and $P\left(z_{i}^{A}\right)$ are only strongly correlated if the combination $y^{B}=m, z^{B}=o$ and $z^{A}=i$ would result in an accident: In that case, $y^{B}=m, z^{B}=o$ has a low probability if $z^{A}=i$ would have high probability and vice versa, as the probability of an accident is unlikely. This effect is the more important, the longer the prediction horizon is chosen. This is because the probabilistic reachable sets overlap at 
the end of long prediction intervals, such that accident situations arise in the overlapping area, see Fig. 5. The exact probability $P\left(y_{m}^{B}, z_{i}^{A}, z_{o}^{B}\right)$ within the overlapping areas is lower than the computed probability $P\left(y_{m}^{B}, z_{o}^{B}\right) P\left(z_{i}^{A}\right)$ due to the assumption of independency.

Next, the joint probability $P\left(y_{j}^{A}, z_{i}^{A}\right)$ is computed from $P\left(y_{j}^{A}, y_{m}^{B}, z_{i}^{A}, z_{o}^{B}\right)$ by

$$
\begin{aligned}
P\left(y_{j}^{A}, z_{i}^{A}\right) & =\sum_{m} \sum_{o} P\left(y_{j}^{A}, y_{m}^{B}, z_{i}^{A}, z_{o}^{B}\right) \\
& =\sum_{m} \sum_{o} P\left(y_{j}^{A} \mid y_{m}^{B}, z_{i}^{A}, z_{o}^{B}\right) P\left(y_{m}^{B}, z_{o}^{B}\right) P\left(z_{i}^{A}\right) \\
& \stackrel{(2)}{=} \sum_{m} \sum_{o} \Omega_{i o}^{j m} p_{o}^{B, m} p_{i}^{A}=\sum_{m}\left[\Omega^{j m} p^{B, m}\right]_{i} p_{i}^{A},
\end{aligned}
$$

as $y_{m}^{B}, z_{o}^{B}$ are mutually exclusive events. After defining the vector

$$
M^{j}:=\sum_{m} \Omega^{j m} p^{B, m}
$$

which is referred to as the selection vector in the following, one can rewrite the above statement as:

$$
P\left(y_{j}^{A}, z_{i}^{A}\right)=M_{i}^{j} p_{i}^{A}=: p_{i}^{A, j},
$$

or in matrix notation:

$$
p^{A, j}=\operatorname{diag}\left(M^{j}\right) p^{A} .
$$

In (1), the joint probability is computed by $p^{j}(k T)=$ $q_{j}([k T,(k+1) T]) p(k T)$. Replacing this term by the result obtained with the interaction matrix $p^{A, j}(k T)=$ $\operatorname{diag}\left(M^{j}(k T)\right) p^{A}(k T)$ allows to extend (1), such that interaction between two vehicles is considered given by:

$$
\begin{aligned}
M^{j}(k T) & =\sum_{m} \Omega^{j m} p^{B, m}(k T), \\
p^{A}((k+1) T) & =\sum_{j} \Phi^{j}(T) \operatorname{diag}\left(M^{j}(k T)\right) p^{A}(k T), \\
p^{A}([k T,(k+1) T]) & =\sum_{j} \Phi^{j}([0, T]) \operatorname{diag}\left(M^{j}(k T)\right) p^{A}(k T) .
\end{aligned}
$$

Equation (3) is the only equation that has to be computed online in order to obtain the stochastic reachable sets of the interacting traffic participants. It is further emphasized, that interaction is not considered in a global manner, i.e. for each vehicle a single probability distribution of possible acceleration inputs is computed. As shown by the derivations for (3), interaction is considered by computing different probabilities of acceleration intervals for each discrete state of the traffic participants.

\section{Interaction on Lanes and Intersections}

The interaction results for two vehicles driving on a lane are extended to two scenarios in this subsection: interaction of several vehicles driving on a lane and interaction at intersections. Interaction on a lane is extended straightforward from two to more vehicles by defining a leading vehicle. The leading vehicle is defined as the vehicle that has the shortest traveling distance to the border of the considered road network. As the leading vehicle cannot orient its velocity based on a vehicle driving ahead, an input probability distribution for free traffic flow $q_{\text {free }}$ is defined and equation (1) is executed. Each vehicle that drives behind the leading vehicle interacts with the vehicle driving ahead, such that for these vehicles (3) is applied.

The interaction at intersections is more complex and modeled in different ways for vehicles that have right of way and those that have not. The reachable set on lanes having right of way are computed as described for the interaction on a lane. The reachable set for the vehicles that do not have right of way are computed in two phases (modes): approaching and crossing. The interaction at intersections is discussed for a simple example with two vehicles $A$ and $B$ as depicted in Fig. 4. Note, that for each vehicle only one path is considered for simplicity. This setup means no loss of generality, as further combinations of driving paths and settings with further vehicles are computed the same way.

The mode approaching is active if the vehicle cannot evaluate the traffic situation due to a limited region of visibility ${ }^{3}$ of the intersection, see Fig. 4 where the approaching vehicle $B$ is labeled by a question mark as it cannot be detected by vehicle $A$. In approaching mode, a virtual vehicle $V$ with zero velocity is put on the paths that do not have right of way where a path with right of way is crossing. This point is also referred to as the crossing position $s_{\text {cross }}$. As a consequence of the virtual vehicle, approaching vehicles (vehicle $A$ in Fig. 4) are forced to decrease their speed when approaching a crossing. As soon as the approaching vehicle $A$ has a sufficiently large region of visibility, the mode crossing is active, see Fig. 4. In mode crossing, the virtual vehicle $V$ is replaced by a probabilistic virtual vehicle $p V$. This vehicle has also zero velocity, but its presence is modeled by a certain probability $p^{V}$. This probability is derived from the probability that vehicle $B$ will cross the path within a certain time interval $\xi \cdot T$. Therefor a set of indices $\mathbb{B}=\left\{i \mid X_{i} \leq s_{\text {cross }}\right\}$ is defined for which the cells $X_{i}$ of vehicle $B$ have position intervals with lower values than the crossing position $s_{\text {cross }}$. The crossing probability of car $B$ in the time interval $t \in[(k-\xi) T, k T]$ is obtained by:

$$
p^{V}(k T)=\sum_{b \in \mathbb{B}} p_{b}^{B}((k-\xi) T)-p_{b}^{B}(k T), \quad k, \xi \in \mathbb{N}^{+} .
$$

The crossing procedure has to be enhanced, when several vehicles that do not have right of way, approach a crossing. It is clear, that these cars have to consider the car driving ahead and the virtual cars $V / p V$ simultaneously. In this case, only actions are allowed that do not result in a crash with neither the car ahead nor the virtual car. The selection vector due to the car driving ahead is denoted $M^{A}$ and the one due to the virtual car representing the crossing traffic is denoted $M^{V}$. The elementwise multiplication $M_{i}^{*}=M_{i}^{A} M_{i}^{V}$ results in an overall selection matrix considering both situations, because the nonzero elements are non-zero elements in $M^{A}$ and $M^{V}$.

\footnotetext{
${ }^{3}$ Region of the road network that can be seen.
} 
Thus, only behavior that is allowed by consideration of both cars is executed. As $\sum_{i} M_{i}^{*} \leq 1$, the overall selection matrix has to be normalized:

$$
M_{i}=\frac{M_{i}^{A} M_{i}^{V}}{\sum_{i} M_{i}^{A} M_{i}^{V}}
$$

It is remarked that the region, in which a vehicle transitions from approaching to crossing mode (see Fig. 4) is in general not known exactly or is not known at all. In the latter case, one can assume a default region that is e.g. 10 meters away from the intersection.

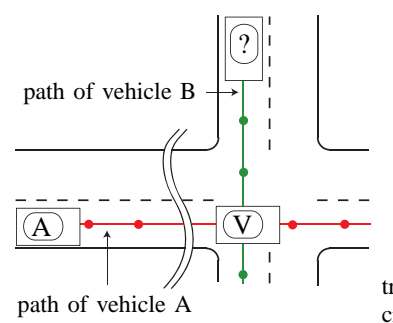

approaching mode

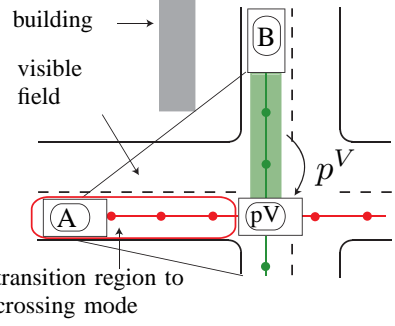

crossing mode
Fig. 4. Intersection scenario: Approaching and crossing mode.

\section{NuMERICAL EXAMPLES}

A numerical example for a lane following scenario and an intersection scenario is given. In the lane following scenario, three cars drive on a single lane, see Fig. 5(a). The stochastic reachable sets can be found in Fig. 5(b)-5(e) of each car for cars driving in one lane (separated into three only for illustration). Each subfigure shows the stochastic reachable sets for a certain time interval and the cars $A, B$ and $C$ are displayed from left to right. The table determining the acceleration interval probabilities based on the number of collision-free acceleration intervals (as presented in Tab. I) is specified as follows for this scenario:

\begin{tabular}{l|llllll}
\hline $\begin{array}{l}\text { collision-free } \\
\text { acc. intervals } h\end{array}$ & $q_{1}^{A}$ & $q_{2}^{A}$ & $q_{3}^{A}$ & $q_{4}^{A}$ & $q_{5}^{A}$ & $q_{6}^{A}$ \\
\hline 6 & 0.05 & 0.05 & 0.1 & 0.3 & 0.4 & 0.1 \\
5 & 0.05 & 0.05 & 0.2 & 0.5 & 0.2 & 0 \\
4 & 0.05 & 0.15 & 0.6 & 0.2 & 0 & 0 \\
3 & 0.1 & 0.6 & 0.3 & 0 & 0 & 0 \\
2 & 0.4 & 0.6 & 0 & 0 & 0 & 0 \\
1 & 1 & 0 & 0 & 0 & 0 & 0 \\
0 & 1 & 0 & 0 & 0 & 0 & 0 \\
\hline
\end{tabular}

The acceleration interval $q_{1}^{A}$ encloses the highest negative acceleration and is gradually increasing until $q_{6}^{A}$ which encloses the highest positive acceleration values. In order to show interaction on a single lane, the leading car $A$ brakes, such that the following cars $B$ and $C$ are forced to brake as well. This can be clearly observed by the mean velocities $\bar{v}$ of the cars for different time intervals, shown on top of Fig. 5(b)-5(e). The labeling on the left side of each subfigure shows the distance on the lane to the origin in meters. Although the stochastic reachable sets of cars $A, B$ and $C$ intersect, the probability that two cars are in the intersection area at the same time is low. This is because the behavior of the traffic participants is restricted to collision-free driving in this work. The computation time is 1.4 seconds for 16 seconds in real time performed with Matlab using a single core desktop computer with $3.7 \mathrm{GHz}$.

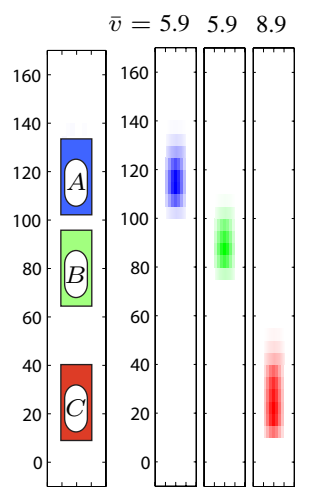

(a) Setup

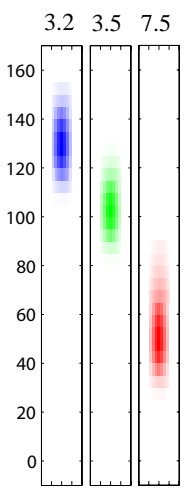

(c) $t=4-8 \mathrm{sec}$

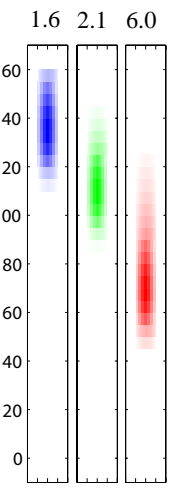

(d) $\mathrm{t}=8-12 \mathrm{sec}$

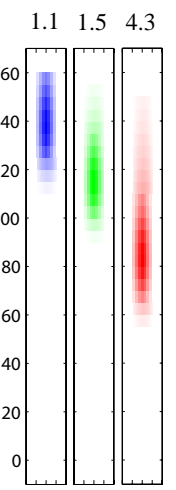

(e) $t=12-16 \mathrm{sec}$

Fig. 5. Reachable sets of the braking scenario.

The intersection scenario consists of an intersection as depicted in Fig. 6(a) showing the initial setting of the scenario. The cars $A$ and $B$ approach the crossing from a street that has no right of way while the cars $C$ and $D$ have right of way. In Fig. 6(b)-6(d), one can see the stochastic reachable sets for selected time intervals. It is clearly recognizable, that cars $A$ and $B$ decrease their velocity when they approach the crossing and that they wait until the crossing cars have passed. The probability that the cars $C$ and $D$ will cross within the next $\xi \cdot T$ seconds is plotted in Fig. 7. The required crossing time $\xi \cdot T$ for the cars $A$ and $B$ is set to 2 seconds for this scenario and is used to determine the probability that the cars $C$ and $D$ cross within the next $\xi \cdot T$ seconds, see (4). The computation time of this scenario is 1.1 seconds for 12.5 seconds in real time using the same software and computer as for the previous example.

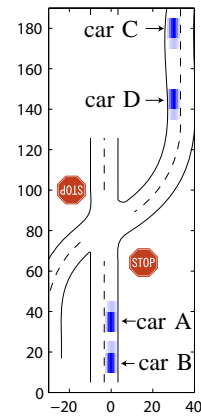

(a) $\mathrm{t}=0 \mathrm{sec}$

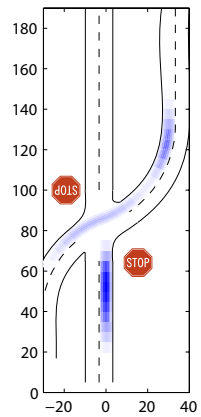

(b) $t=3.5-4 \mathrm{sec}$
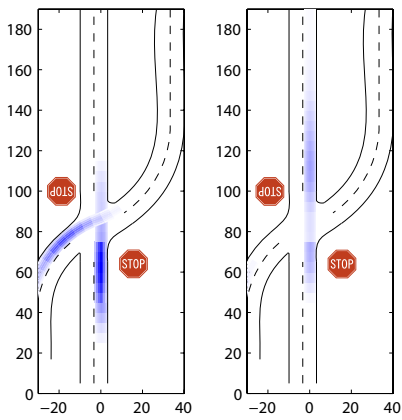

(c) $\mathrm{t}=7-7.5 \mathrm{sec}$ (d) $t=10.5-11 \mathrm{sec}$

Fig. 6. Reachable sets of the intersection scenario

\section{CONCLUSION}

A method for the computation of stochastic reachable sets of interacting traffic participants has been presented. The 


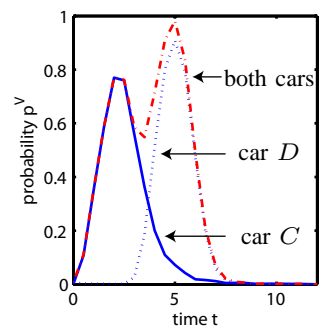

Fig. 7. Probability of crossing cars for the intersection scenario.

approach is efficient enough to be implemented online when several traffic participants are regarded. The approach can be extended to multi-lane traffic where traffic participants can change lanes. A sketch of the extension is the following: A vehicle $A$ is changing to lane $e$ with high probability, if it would not crash into a vehicle $B$ driving ahead on lane $e$ and if another vehicle $C$ that would follow on lane $e$ after the lane change, is not forced to brake. The probability, that vehicle $C$ has to brake on lane $e$ when vehicle $A$ is driving ahead after the lane change, can be obtained by summation of the selection vector $M$ of vehicle $C$.

\section{ACKNOWLEDGMENTS}

The authors gratefully acknowledge partial support of this work by the Deutsche Forschungsgemeinschaft (German Research Foundation) within the Transregional Collaborative Research Centre 28 Cognitive Automobiles.

\section{REFERENCES}

[1] A. Vahidi and A. Eskandarian, "Research advances in intelligent collision avoidance and adaptive cruise control," IEEE Transactions on Intelligent Transportation Systems, vol. 4, pp. 143-153, 2003.

[2] J. Hillenbrand, A. M. Spieker, and K. Kroschel, "A multilevel collision mitigation approachits situation assessment, decision making, and performance tradeoffs," IEEE Transactions on Intelligent Transportation Systems, vol. 7, pp. 528-540, 2006.

[3] T. Hesse and T. Sattel, "An approach to integrate vehicle dynamics in motion planning for advanced driver assistance systems," in Proc. of the IEEE Intelligent Vehicles Symposium, 2007, pp. 1240-1245.

[4] A. Broadhurst, S. Baker, and T. Kanade, "Monte carlo road safety reasoning," in Proc. of the IEEE Intelligent Vehicles Symposium, 2005, pp. 319-324.

[5] A. Eidehall and L. Petersson, "Threat assessment for general road scenes using monte carlo sampling," in Proc. of the Intelligent Transportation Systems Conference, 2006, pp. 1173-1178.

[6] S. Danielsson, L. Petersson, and A. Eidehall, "Monte carlo based threat assessment: Analysis and improvements," in Proc. of the IEEE Intelligent Vehicles Symposium, 2007, pp. 233-238.

[7] M. Prandini and J. Hu, "A stochastic approximation method for reachability computations," Final Report of the Hybridge Project, pp. 115-147, 2005.

[8] J. Hu, M. Prandini, and S. Sastry, "Aircraft conflict detection in presence of a spatially correlated wind field," IEEE Transactions on Intelligent Transportation Systems, vol. 6, pp. 326-340, 2005.

[9] M. Althoff, O. Stursberg, and M. Buss, "Safety assessment of autonomous cars using verification techniques," in Proc. of the American Control Conference, 2007, pp. 4154-4159.

[10] —, "Online verification of cognitive car decisions," in Proc. of the 2007 IEEE Intelligent Vehicles Symposium, 2007, pp. 728-733.

[11] T. Toledo, "Integrated driving behavior modeling," Ph.D. dissertation, Massachusetts Institute of Technology, 2003.

[12] P. Ehlert and L. Rothkrantz, "Microscopic traffic simulation with reactive driving agents," in Proc. of the IEEE intelligent Transportation Systems Conference, 2001, pp. 860-865.
[13] A. E. Broadhurst, S. Baker, and T. Kanade, "A prediction and planning framework for road safety analysis, obstacle avoidance and driver information," in Proc. of the 11th World Congress on Intelligent Transportation Systems, October 2004.

[14] T. Henzinger, The theory of hybrid automata, ser. NATO ASI Series F: Computer and Systems Sciences. Springer, 2000, vol. 170, pp. 265-292. 